\title{
Comparison of Outcomes of Percutaneous Coronary Intervention Versus Coronary Artery Bypass Grafting
}

Ratan Kumar ${ }^{1}$, Kheraj Mal ${ }^{2}$, Muhammad Khalid Razaq ${ }^{3}$, Mansoor Magsi ${ }^{4}$, Muhammad Khizar Memon ${ }^{5}$, Sidra Memon ${ }^{6}$, Sana Irfan ${ }^{6}$, Kanwal Bansari ${ }^{6}$, Basma Ali ${ }^{6}$, Amber Rizwan ${ }^{7}$

1. Cardiology, Khairpur Medical College, Nawabshah, PAK 2. Cardiology, National Institute of Cardiovascular Diseases, Sukkur, PAK 3. Cardiology, Sheikh Zayed Medical College, Rahim Yar Khan, PAK 4. Internal Medicine, Taluka Hospital Kandhkot, Kandhkot, Kashmore, PAK 5. Internal Medicine, Liaquat University of Medical and Health Sciences, Hyderabad, PAK 6. Internal Medicine, Jinnah Sindh Medical University, Karachi, PAK 7. Family Medicine, Jinnah Post Graduate Medical Center, Karachi, PAK

Corresponding author: Amber Rizwan, amber_aljazeera109@hotmail.com

\section{Abstract}

\section{Introduction}

Percutaneous coronary intervention (PCI) and coronary artery bypass grafting (CABG) are two common treatment options used in patients suffering from coronary artery disease. Selection and favorability of one over the other depend on individual clinical scenarios. The purpose of this study is to compare outcomes after treatment with PCI and CABG.

\section{Methods}

This longitudinal observational study was conducted from April 2018 to July 2019 in a cardiovascular unit of a tertiary care hospital. Participants who were eligible for revascularization were randomized either to receive stent $(\mathrm{PCI})$ or surgery (CABG). Patients were then followed up for 12 months for the development of all-cause mortality and major adverse cardiovascular events (MACEs).

\section{Results}

At 12 months, patients randomized to the PCI group had an increased risk of repeat revascularization (21.3\% vs. $7.4 \%$; $=0.007$ ), whereas a similar number of patients in both groups died $(3.8 \%$ vs. $3.7 \%)$, suffered myocardial infarction (7.6\% vs. $5.6 \%$ ), or had a cerebrovascular accident ( $3.8 \%$ vs. $2.8 \%$ ).

\section{Conclusions}

This study showed that PCI had an increased risk of repeat revascularization compared to CABG. However, both had comparable significance in the development of MACEs. Nevertheless, there is a need for further study to better assess the outcomes of either, especially in the long run.

Review began 12/05/2020 Review ended 12/08/2020 Published 12/21/2020

\section{() Copyright 2020}

Kumar et al. This is an open access article distributed under the terms of the Creative Commons Attribution License CC-BY 4.0., which permits unrestricted use, distribution, and reproduction in any medium, provided the original author and source are credited.
Categories: Cardiac/Thoracic/Vascular Surgery, Cardiology, Internal Medicine

Keywords: percutaneous coronary intervention, coronary artery bypass grafting, myocardial infarction

\section{Introduction}

Currently, both percutaneous coronary intervention (PCI) and coronary artery bypass grafting (CABG) are reliable therapeutic options for patients with left main coronary disease. Treatment selection should consider the extent of the disease (favoring CABG for severe lesions) and patient preference (usually favoring PCI) [1]. The clinical efficacy of PCI has been established in acute ischemia and in other limited situations. However, accumulating evidence shows that patients with complex atherosclerotic lesions, multivessel disease, left main stem disease, left ventricular dysfunction (LVD), and diabetes mellitus (DM) derive more benefit from CABG than from PCI. Therefore, CABG should be presented to these patients as the more effective intervention in terms of survival, freedom from re-intervention, and cost-effectiveness [2]. However, patients prefer not to undergo surgery because of a fear of complications and of physical and mental debilitation because of surgery [3].

In patients deemed inoperable for CABG with a high-risk profile, a suboptimal outcome was achieved after PCI. In patients who are not candidates for PCI, bypass surgery produces excellent results [4]. Kappetein et al. found after three-year follow-up that patients with more complex disease have an increased risk of an allcause mortality and major adverse cardiovascular events (MACEs) with PCI, and CABG is the preferred treatment option [5]. In left main coronary artery disease (CAD), CABG reduced major adverse cardiac or cerebrovascular events at five years compared with PCI with drug-eluting stents [6]. In a randomized controlled trial (RCT), after 10 years, CABG in older patients was associated with fewer subsequent coronary 


\section{Cureus}

interventions, whereas PCI treatment was associated with a higher incidence of myocardial infarction (MI) [7].

The need to assess outcomes of PCI and CABG is vital in appropriate treatment selection according to each individual's relevant conditions and to better understand which modality will result in better outcomes that may help to improve the quality of life and mortality of these patients in the future.

\section{Materials And Methods}

This longitudinal observation study was conducted from April 2018 to July 2019 in a cardiovascular unit of a tertiary care hospital. A total of 220 participants were randomized by 1:1 ratio using an online software Research Randomizer (https://www.randomizer.org) to either receive stent through PCI ( $\mathrm{n}=103)$ or to undergo CABG $(\mathrm{n}=107)$. The procedures were explained to each participant. Inclusion criteria included patients with silent, stable, or unstable angina, and the presence of at least two lesions in different coronary arteries, eligible for revascularization. All patients or their attendants gave written, informed consent.

Patients were followed up for 12 months or till the development of all-cause mortality and MACEs, whichever came first. In this study, MACEs were defined as death, cerebrovascular accident, MI, and revascularization. Overall, 10 patients were lost to follow-up; seven from the PCI group and three from the CABG group. Patients who completed the study were included in the final analysis.

Statistical analysis was performed using SPSS version 23.0 (IBM Corp., Armonk, NY, USA). Continuous variables were presented as mean and SD. Binary outcomes were expressed as frequencies and percentages and were compared in terms of relative risk with $95 \% \mathrm{CI}$.

\section{Results}

Both groups, PCI and CABG, had a similar demographic and risk factor profile (Table 1).

\begin{tabular}{|c|c|c|}
\hline Characteristics & $\mathrm{PCl}(n=103)$ & CABG $(n=107)$ \\
\hline Age (years) & $59 \pm 9$ & $59 \pm 10$ \\
\hline Male, \% & 62 & 61 \\
\hline Diabetes, \% & 20 & 21 \\
\hline Hypertension, \% & 44 & 43 \\
\hline Hypercholesterolemia, \% & 61 & 62 \\
\hline Current smoker, \% & 25 & 25 \\
\hline Previous MI, \% & 31 & 32 \\
\hline Unstable angina, \% & 32 & 31 \\
\hline
\end{tabular}

\section{TABLE 1: Comparison of demographic data and risk factor profile between PCI and CABG}

CABG, coronary artery bypass grafting; $\mathrm{MI}$, myocardial infarction; $\mathrm{PCI}$, percutaneous coronary intervention.

At 12 months, patients randomized to the PCI group had an increased risk of repeat revascularization (21.3\% vs. $7.4 \%, \mathrm{p}=0.007$ ), whereas a similar number of patients in both groups died, or suffered MI or cerebrovascular accident (Table 2). 


\section{Cureus}

\begin{tabular}{|c|c|c|c|c|}
\hline Event & $\mathrm{PCI}(\mathrm{n}=103)$ & CABG $(n=107)$ & Relative risk (95\% Cl) & p-Value \\
\hline Death & $4(3.8 \%)$ & $4(3.7 \%)$ & $1.03(0.26-4.04)$ & NS \\
\hline Cerebrovascular accident & $4(3.8 \%)$ & $3(2.8 \%)$ & $1.38(0.31-6.03)$ & NS \\
\hline Myocardial infarction & $8(7.6 \%)$ & $6(5.6 \%)$ & $1.35(0.48-3.78)$ & NS \\
\hline Repeat revascularization & $22(21.3 \%)$ & $8(7.4 \%)$ & $2.85(1.33-6.12)$ & 0.007 \\
\hline
\end{tabular}

\section{TABLE 2: Comparison of outcomes between $\mathrm{PCI}$ and CABG}

CABG, coronary artery bypass grafting; NS, nonsignificant; PCI, percutaneous coronary intervention.

\section{Discussion}

PCI and CABG are considered revascularization procedures, but only CABG has been proven to provide longterm prognostic benefits in patients with $\mathrm{CAD}[2,8]$. Myocardial infarcts are usually generated by non-flowlimiting stenosis. PCI is mainly focused on treating flow-limiting stenosis, and thus it cannot limit the formation of new infarcts. CABG, on the other hand, can significantly limit the formation of new infarcts by providing flow distal to the vessel occlusion [8].

This study was conducted to assess the outcomes of PCI and CABG in patients with silent, stable, or unstable angina, and the presence of at least two lesions in different coronary arteries eligible for revascularization. Patients in both groups (PCI and CABG) had similar demographic and risk factor profile including age (59 \pm 9 vs. $59 \pm 10$ ), male gender ( $62 \%$ vs. $61 \%$ ), DM ( $20 \%$ vs. $21 \%$ ), hypertension ( $44 \%$ vs. $43 \%$ ), hypercholesterolemia (61\% vs. $62 \%$ ), present smoking history ( $25 \%$ vs. $25 \%$ ), history of MI (31\% vs. $32 \%$ ), and unstable angina (32\% vs 31\%). After 12 months of follow-up, patients in the PCI group had an increased risk of repeat revascularization $(22.1 \%$ vs. $7.4 \%, p=0.007)$ as compared to patients in the CABG group. However, MACEs including mortality rate and incidence of both MI and cerebrovascular accident were similar in both groups in our study. Similar findings were reported by Palmerini et al., who concluded that both PCI and CABG had similar long-term composite rates of mortality, MI, and cerebrovascular disease, with PCI demonstrating early 30-day safety advantage and CABG procedure showing lesser rates of unplanned revascularization [9].

Rosenfeldt et al. in their study analyzed recent evidence to conclude that CABG is more beneficial than PCI in patients with complex atherosclerotic lesions, multivessel disease, left middle circumflex artery (LMCA) disease, LVD, and DM. However, patients still usually prefer PCI because the procedure is minimally invasive [2]. In a systemic review that included 13 RCTs and five meta-analyses to demonstrate the effectiveness of PCI and CABG, Deb et al. concluded that CABG should be recommended in patients with DM, LMCA disease, multivessel CAD, or LVD, and SYNTAX score $>22$ (severe CAD) due to improved long-term survival and lower cardiac events, whereas PCI is considered in patients with less complex CAD (SYNTAX $\leqslant 22$ ) or if the patient is at a high surgical risk [10]. Patients with multivessel involvement or LMCA reported greater relief from angina after six and 12 months of undergoing CABG compared to PCI [11].

The study has its limitations. It was a single-center study and hence sample size diversity was reduced. Patients were not followed up for a longer duration, as most of them came from the peripheral area and there was a strong chance that they might be lost to follow-up. As our study was limited to a 12-month follow-up, there is room to investigate outcomes over a longer period of time to further learn about the consequences of PCI and CABG in the long run. To the best of our knowledge, this is the first local study comparing the outcome of PCI vs. CABG. It will add to the limited local data we have related to cardiovascular outcomes associated with two techniques and assist clinicians in making an informed decision.

\section{Conclusions}

It can be concluded that PCI could lead to an increased risk of revascularization compared to CABG. However, there is no superiority of one over another in terms of having a risk of MI, cerebrovascular accident, or death after PCI or CABG. These findings suggest CABG to be slightly more favorable, but it highly depends on the patient's consent, requirement, and severity of the disease.

\section{Additional Information \\ Disclosures}

Human subjects: Consent was obtained by all participants in this study. Khairpur Medical College issued approval KMC/18/IRB-Off/61. Animal subjects: All authors have confirmed that this study did not involve 
animal subjects or tissue. Conflicts of interest: In compliance with the ICMJE uniform disclosure form, all authors declare the following: Payment/services info: All authors have declared that no financial support was received from any organization for the submitted work. Financial relationships: All authors have declared that they have no financial relationships at present or within the previous three years with any organizations that might have an interest in the submitted work. Other relationships: All authors have declared that there are no other relationships or activities that could appear to have influenced the submitted work.

\section{References}

1. Morice MC: Patients with left main coronary artery disease: stent or surgery? . Lancet. 2020, 395:167-8. 10.1016/S0140-6736(19)33224-6

2. Rosenfeldt FL, Wilson MD, Buxton BF, Marasco SF: Coronary artery bypass surgery provides long-term results superior to percutaneous coronary intervention. Heart Lung Circ. 2012, 21:1-11. 10.1016/j.hlc.2011.08.008

3. Kawasuji M: Debate over patient-centered care: percutaneous coronary intervention or coronary artery bypass grafting?. Surg Today. 2011, 41:459-62. 10.1007/s00595-010-4452-4

4. Head SJ, Holmes DR Jr, Mack MJ, et al.: Risk profile and 3-year outcomes from the SYNTAX percutaneous coronary intervention and coronary artery bypass grafting nested registries. JACC Cardiovasc Interv. 2012, 5:618-25. 10.1016/j.jcin.2012.02.013

5. Kappetein AP, Feldman TE, Mack MJ, et al.: Comparison of coronary bypass surgery with drug-eluting stenting for the treatment of left main and/or three-vessel disease: 3-year follow-up of the SYNTAX trial. Eur Heart J. 2011, 32:2125-34. 10.1093/eurheartj/ehr213

6. Bates ER: In left main CAD, CABG reduced major adverse cardiac or cerebrovascular events at 5 years compared with PCI. Ann Intern Med. 2017, 166:20. 10.7326/ACPJC-2017-166-4-020

7. Rezende PC, Hueb W, Garzillo CL, Lima EG, Hueb AG, Ramires JAF, Filho RK: Ten-year outcomes of patients randomized to surgery, angioplasty, or medical treatment for stable multivessel coronary disease: effect of age in the Medicine, Angioplasty, or Surgery Study II trial. J Thorac Cardiovasc Surg. 2013, 146:1105-12. 10.1016/j.jtcvs.2012.08.015

8. Doenst T, Haverich A, Serruys P, et al.: PCI and CABG for treating stable coronary artery disease: JACC review topic of the week. J Am Coll Cardiol. 2019, 73:964-76. 10.1016/j.jacc.2018.11.053

9. Palmerini T, Serruys P, Kappetein AP, et al.: Clinical outcomes with percutaneous coronary revascularization vs coronary artery bypass grafting surgery in patients with unprotected left main coronary artery disease: a meta-analysis of 6 randomized trials and 4,686 patients. Am Heart J. 2017, 190:54-63. 10.1016/j.ahj.2017.05.005

10. Deb S, Wijeysundera HC, Ko DT, Tsubota H, Hill S, Fremes SE: Coronary artery bypass graft surgery vs percutaneous interventions in coronary revascularization: a systematic review. JAMA. 2013, 310:2086-95. 10.1001/jama.2013.281718

11. Cohen DJ, Van Hout B, Serruys PW, et al.: Quality of life after PCI with drug-eluting stents or coronaryartery bypass surgery. N Engl J Med. 2011, 364:1016-26. 10.1056/NEJMoa1001508 